\title{
A New Approach for Segmentation and Classification of Textures by using Square Primitive Pattern
}

\author{
M. Radhika Mani, K. Chandra Shekar \\ ${ }^{1}$ (Assoc. Professor, Dept. of CSE, Pragati Engineering College) \\ ${ }^{2}$ (Asst. Professor, Dept. of CSE, Pragati Engineering College)
}

\begin{abstract}
The Texture classification is an important task in the field of object recognition. The texture classification is highly dependent on the segmentation of objects in the input image. So, the present paper proposes a morphological approach to detect the objects and extract the edges of the detected objects. Then the present paper measures recurrence of the square primitive pattern (SPP) in each of the $3 \times 3$ local window. Based on this measurement, the textures are precisely classified. This is proved by the experimental results conducted on different textures.
\end{abstract}

Keywords: - About five key words in alphabetical order, separated by comma

\section{INTRODUCTION}

Textures are characterized by spatial distributions of image pixel values in a local neighborhood region. Though there is no unique definition of texture because of its wide variability, somehow it can be defined as a structure composed of a large number of more or less ordered similar elements or patterns. Texture classification remains a fundamental task for image processing. Texture features [10] are often used to classify them into predetermined classes. In actual applications of texture classification, it is unlikely that texture samples will be digitized under the same noise levels as the samples used to train the classifier. It would be useful to have texture features that are insensitive to fluctuations in noise level. The problem of edge or boundary detection from images is critical in computer vision and has been largely studied. Popular existing techniques can be classified into two families: i) local ones such as edge detectors $[1,2]$ which use image filtering and local information and ii) active contours techniques like "snakes" or "balloons" based on information along the boundaries [3, 4]. The local methods are able to high light the main frontiers of the image. The active contours provide better results to capture reliable frontiers but require a good initialization of the initial snake. The present paper uses local edge detection technique by enhanced filter and local information. Study of patterns on textures is recognized as an important step in characterization and classification of texture [11]. It has been used to filter out speckle in radar data [5] and to remove the effects of regular agricultural patterns in image data [5]. Study of regular patterns based on fundamentals of local variance was carried out recently [6]. Hence, the study of patterns still plays a significant area of research in classification and characterization of textures. That's why the present paper investigates how the frequency of occurrences of patterns varies after applying different random thresholds for binary image of the original textured image. The present paper assumes texture is characterized not only by the gray value at a given pixel, but also by the gray value pattern in a neighborhood surrounding the pixel. The ability to efficiently analyze and describe textured patterns is thus of fundamental importance. A simple or complex pattern of a neighborhood can be considered as one of the texture primitive feature [7]. The present paper proposes a new method for texture classification based on square primitive pattern (SPP) on edges or segments of the edges extracted. The organization of the paper is as follows. The methodology of texture classification by random thresholds and measure of frequencies is presented in section II, the results and discussions are presented in section III and the conclusions are presented in the last section.

\section{METHODOLOGY}

The significant feature of the object is its texture property. So, texture classification is becoming more and more crucial nowadays. The proposed algorithm initially extracts the objects existing in the input gray image by using novel segmentation algorithm. The segmentation algorithm applies various mathematical concepts related to eight connected neighborhood of each pixel. The algorithm for segmentation is given by Algorithm 1.

Algorithm 1: Segmentation of input gray image to extract the objects

Step 1: Read the input image

Step 2: The original input image is preprocessed by a recursive digital filter developed by Modestino and fries [8]. This recursive filter is an isotropic, band pass wiener filter combined with a Laplacian operator for edge 
detection. It is assumed that the image is represented by random variables corrupted by white Gaussian noise of zero mean. The filter output is given by the Equation (1) where $\mathrm{N}_{\mathrm{I}}$ denotes the neighborhood pixel.

$$
\mathrm{O}=\left(-\mathrm{a} \times\left(\mathrm{O}\left(\mathrm{N}_{2}\right)+\mathrm{O}\left(\mathrm{N}_{8}\right)\right)\right)+\left(-\mathrm{b} \times \mathrm{O}\left(\mathrm{N}_{4}\right)\right)+\left(\mathrm{c} \times \mathrm{N} \times \mathrm{I}\left(\mathrm{N}_{2}\right) \times \mathrm{I}\left(\mathrm{N}_{8}\right)\right)+\left(\mathrm{d} \times \mathrm{I}\left(\mathrm{N}_{2}\right)\right)
$$

Step 3: Calculate four sub images by using equations (2)-(5)

$$
\begin{aligned}
& \mathrm{S}_{1}=\mathrm{O}\left(\mathrm{N}_{1}\right)-\mathrm{O}\left(\mathrm{N}_{5}\right) \\
& \mathrm{S}_{2}=\sqrt{2} \times\left[\mathrm{O}\left(\mathrm{N}_{2}\right)-\mathrm{O}\left(\mathrm{N}_{4}\right)\right] \\
& \mathrm{S}_{3}=\mathrm{O}\left(\mathrm{N}_{7}\right)-\mathrm{O}\left(\mathrm{N}_{3}\right) \\
& \mathrm{S}_{2}=\sqrt{2} \times\left[\mathrm{O}\left(\mathrm{N}_{2}\right)-\mathrm{O}\left(\mathrm{N}_{6}\right)\right]
\end{aligned}
$$

Step 4: Obtain the segmented image from the four generated sub images with equation (6).

$$
\mathrm{V}=\left\{\begin{array}{l}
1 \text { if }\left(\left|\max \left(\mathrm{S}_{1}, \mathrm{~S}_{2}, \mathrm{~S}_{3}, \mathrm{~S}_{4}\right)\right|^{2}-\left(\mathrm{F} \times \mathrm{O}^{2}\right)\right)>=\mathrm{T}_{\mathrm{e}} \\
0 \text { else }\left(\left(\left.\max \left(\mathrm{S}_{1}, \mathrm{~S}_{2}, \mathrm{~S}_{3}, \mathrm{~S}_{4}\right)\right|^{2}-\left(\mathrm{F} \times \mathrm{O}^{2}\right)\right)<\mathrm{T}_{\mathrm{e}}\right.
\end{array}\right.
$$

After extracting the segments from the image, the next step is to measure the frequency occurrence of various patterns in the segments identified. Depending on the context the word pattern has many different interpretations. The biology community seems to use the word pattern without defining it. The implicit meaning generally brings to mind some kind of repeated arrangement (regular or not) and the term is often defined by examples. The word texture certainly has many interpretations in the graphics community. Using a $3 \times 3$ grid one can generate 512 patterns. However, if we specify the center point of a $3 \times 3$ grid should be a grain component then the number of spatial patterns will be reduced to 256 . The present study uses this concept. It is possible to enumerate all the 256 patterns using a $3 \times 3$ grid. But such an exhaustive enumeration is removed in the present paper by considering only 8 simple patterns. The present paper has proposed an approach for classifying textures by using Square Pattern Primitive (SPP). The SPP is given by the Fig. 1.

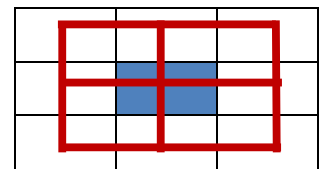

Fig. 1 Structure of SPPP pattern.

The present paper considers SPP pattern when the central pixel is necessarily a grain component. The SPP is decomposed into the occurrence of simple patterns like Top Horizontal Line (THLP), Middle Horizontal Line (MHLP), Bottom Horizontal Line (BHLP), Left Vertical Line (LVLP), Middle Vertical Line (MVLP), Right Vertical Line (RVLP), Left Diagonal (LDP), and Right Diagonal (RDP) are studied. The Fig. 2 specifies the particular kind of arrangement of the above simple patterns.

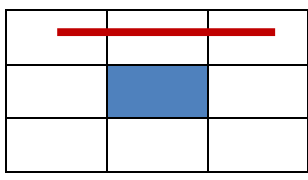

(a)

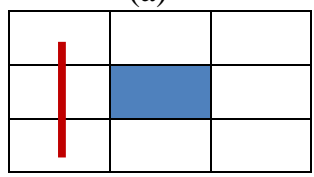

(d)

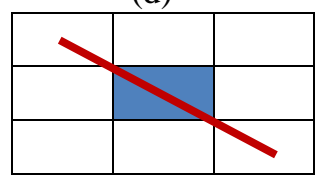

(g)

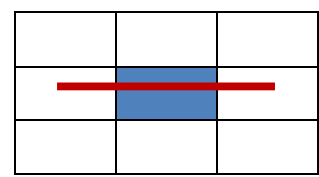

(b)

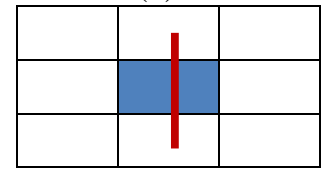

(e)

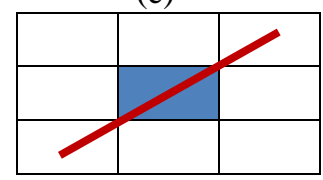

(h)

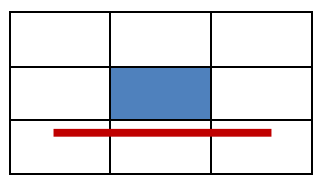

(c)

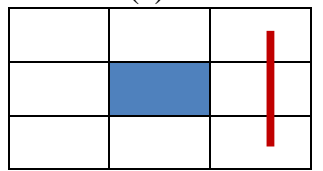

(f)

Fig. 2 Representation of Primitive Patterns (a) THLP (b) MHLP (c) BHLP (d) LVLP (e) MVLP (f) RVLP (g) LDP (h) RDP. 


\section{RESULTS AND DISCUSSIONS}

The present paper has considered 48 textures from visitex album, 8 granite textures and 8 marble textures from the website [9] and Brodatz textures with a resolution of $256 \times 256$. The values of the parameters used in the segmentation algorithm are $a=-0.3602, b=-0.154, c=-0.8264, d=0.0220, F=0.2$ and $T_{e}=1$. The step by step results of segmentation are given in Fig. 3 to 6 . From these results, it is clear that the enhancement filter brightens the edge part of the objects existing in the input image. Then the first generated sub image strengthens vertical edges of the enhanced result. The second sub image clearly extracts only horizontal edges. The third sub image strengthens the horizontal sub image and the fourth sub image extracts only vertical edges. Finally the segmented image extracts the region in between horizontal and vertical edges extracted.

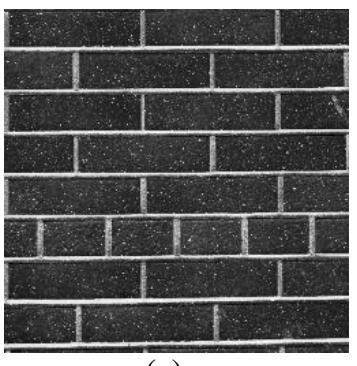

(a)

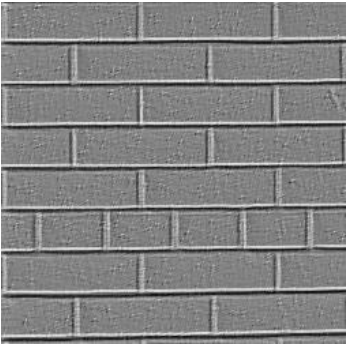

(e)

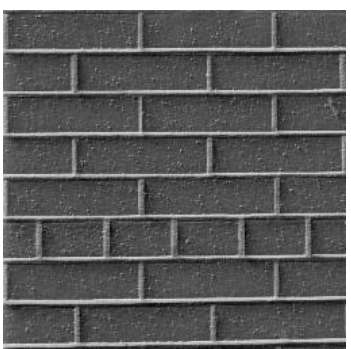

(b)

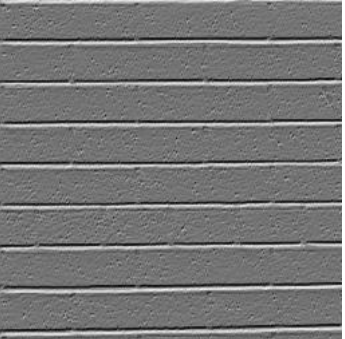

(f)

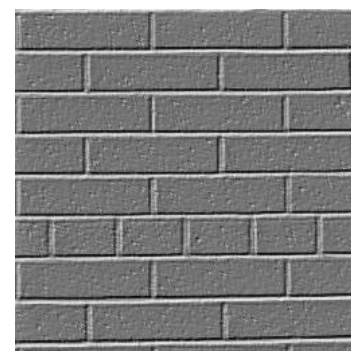

(c)

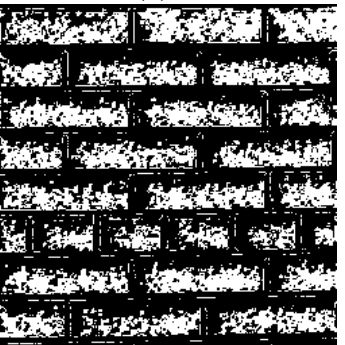

(g)

Fig. 3 (a) Original Image - Brodatz D26 (b) Enhancement Filter (c) First Sub Image (d) Second Sub Image (e) Third Sub Image (f) Fourth Sub Image (g) Segmented Image.

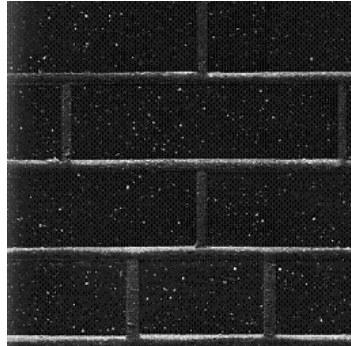

(a)

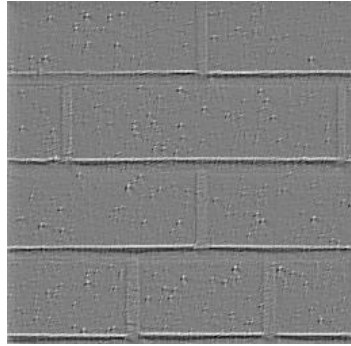

(e)

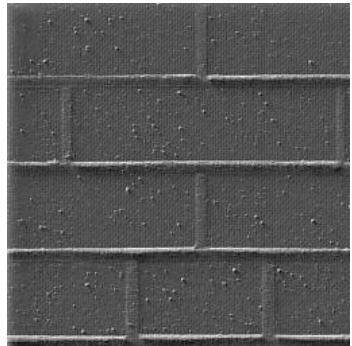

(b)

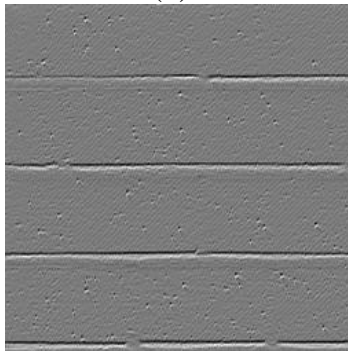

(f)

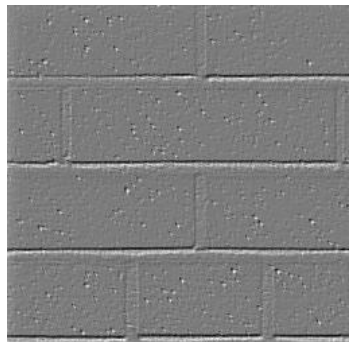

(c)

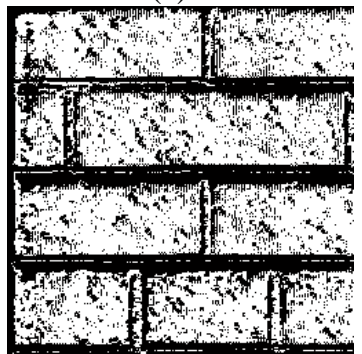

(g)

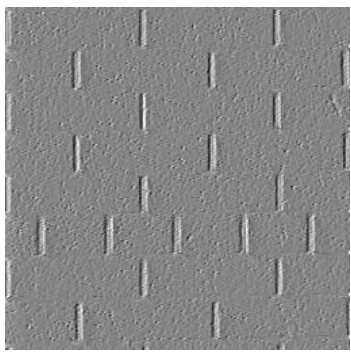

(d)

Fig. 4 (a) Original Image - Brodatz D25 (b) Enhancement Filter (c) First Sub Image (d) Second Sub Image (e) Third Sub Image (f) Fourth Sub Image (g) Segmented Image. 


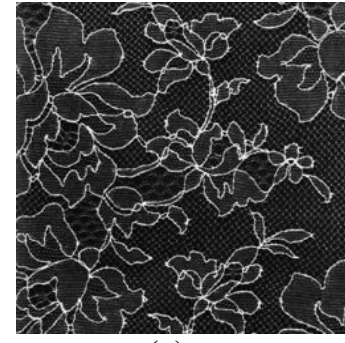

(a)

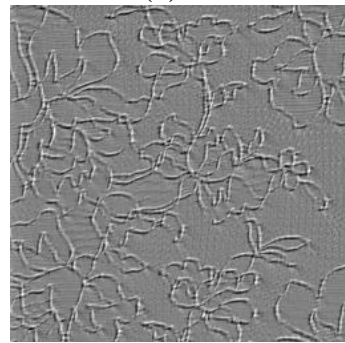

(e)

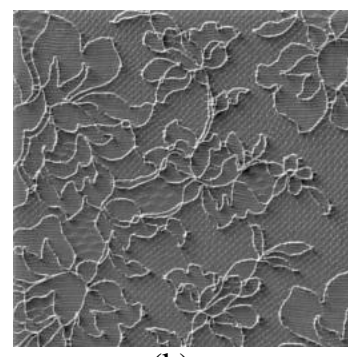

(b)

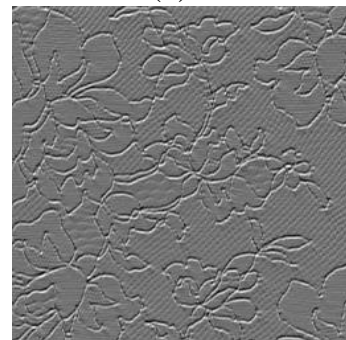

(f)

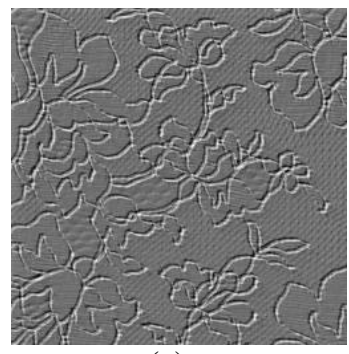

(c)

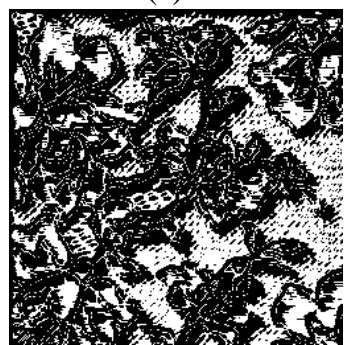

(g)

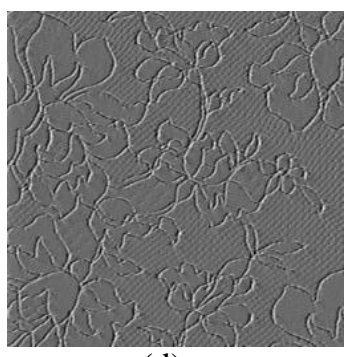

(d)

Fig. 5 (a) Original Image - Brodatz D41 (b) Enhancement Filter (c) First Sub Image (d) Second Sub Image (e) Third Sub Image (f) Fourth Sub Image (g) Segmented Image.

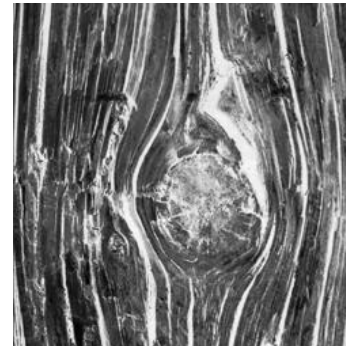

(a)

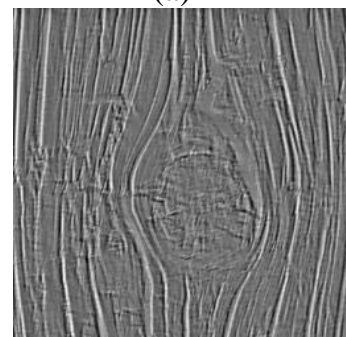

(e)

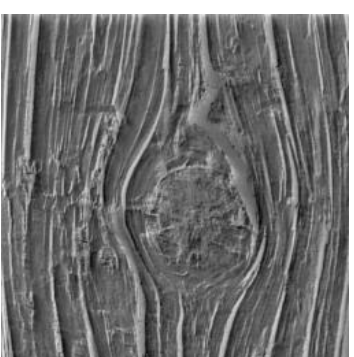

(b)

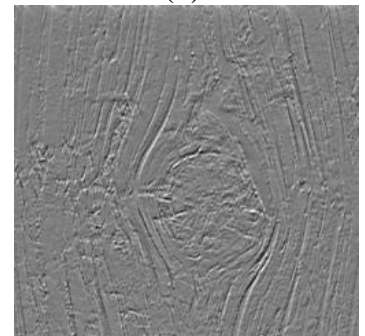

(f)

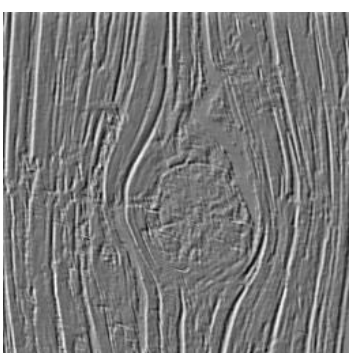

(c)

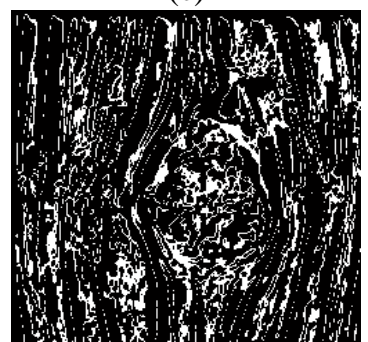

(g)

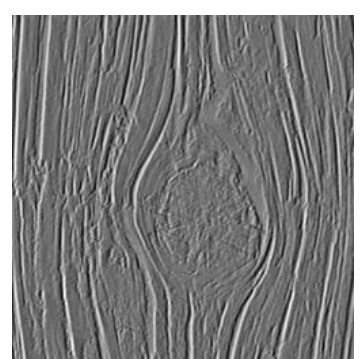

(d)

Fig. 6 (a) Original Image - Brodatz D72 (b) Enhancement Filter (c) First Sub Image (d) Second Sub Image (e) Third Sub Image (f) Fourth Sub Image (g) Segmented Image.

The present method calculated average occurrence of horizontal lines, vertical lines and diagonal lines as denoted by AOHL, AOVL and AODL respectively by the Equations (7)- (8) on a given window size. Table 1,2 and 3 indicates average occurrence of various primitive patterns of various textures obtained on the subimages of the given textures. The Fig. (7)-(12) clearly indicates a good discrimination of various textures.

$$
\begin{aligned}
& A O H L=\frac{(T H L+M H L+B H L)}{3} \\
& A O V L=\frac{(L V L+M V L+R V L)}{3} \\
& A O D L=\frac{(L D+R D)}{2}
\end{aligned}
$$


Table 1 Average occurrence of various primitive patterns of Brick Texture

\begin{tabular}{|c|c|c|c|c|}
\hline Texture No. & Texture Name & AOHL & AOVL & AODL \\
\hline 1 & Brick.0000 & 27.83 & 24.81 & 22.15 \\
\hline 2 & Brick.0002 & 64.95 & 58.69 & 59.02 \\
\hline 3 & Brick.0003 & 48.86 & 44.87 & 44.27 \\
\hline 4 & Brick.0004 & 31.68 & 30.82 & 29.07 \\
\hline 5 & Brick.0005 & 34.03 & 34.95 & 32.49 \\
\hline 6 & Brick.0006 & 27.17 & 28.30 & 25.91 \\
\hline 7 & Brick.0007 & 47.81 & 47.51 & 46.73 \\
\hline 8 & Brick.0008 & 45.95 & 45.39 & 44.43 \\
\hline
\end{tabular}

Table2. Average occurrence of various primitive patterns of Fabric Texture

\begin{tabular}{|c|c|c|c|c|}
\hline Texture No. & Texture Name & AOHL & AOVL & AODL \\
\hline 1 & Fabric.0000 & 39.42 & 40.25 & 39.30 \\
\hline 2 & Fabric.0004 & 48.80 & 42.50 & 45.42 \\
\hline 3 & Fabric.0007 & 26.62 & 28.74 & 26.57 \\
\hline 4 & Fabric.0008 & 72.63 & 72.61 & 74.46 \\
\hline 5 & Fabric.0011 & 39.35 & 39.39 & 39.28 \\
\hline 6 & Fabric.0013 & 66.83 & 63.60 & 64.97 \\
\hline 7 & Fabric.0015 & 47.64 & 50.25 & 47.92 \\
\hline 8 & Fabric.0018 & 19.81 & 18.96 & 19.03 \\
\hline
\end{tabular}

Table 3. Average occurrence of various primitive patterns of Water Texture

\begin{tabular}{|c|c|c|c|c|}
\hline Texture No. & Texture Name & AOHL & AOVL & AODL \\
\hline 1 & Water.0000 & 57.26 & 49.24 & 51.13 \\
\hline 2 & Water.0001 & 10.22 & 9.51 & 9.42 \\
\hline 3 & Water.0002 & 25.79 & 20.13 & 19.91 \\
\hline 4 & Water.0003 & 21.14 & 14.50 & 14.29 \\
\hline 5 & Water.0004 & 68.08 & 63.49 & 65.11 \\
\hline 6 & Water.0005 & 21.55 & 16.19 & 16.02 \\
\hline 7 & Water.0006 & 17.10 & 12.13 & 11.96 \\
\hline 8 & Water.0007 & 51.79 & 44.35 & 44.73 \\
\hline
\end{tabular}

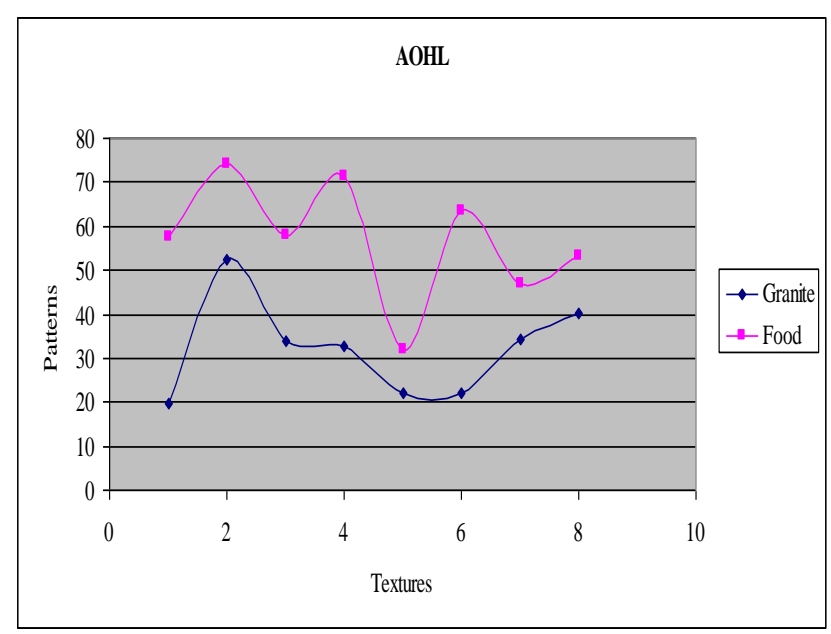

Fig. 7 Classification of Granite and Food Textures by AOHL 


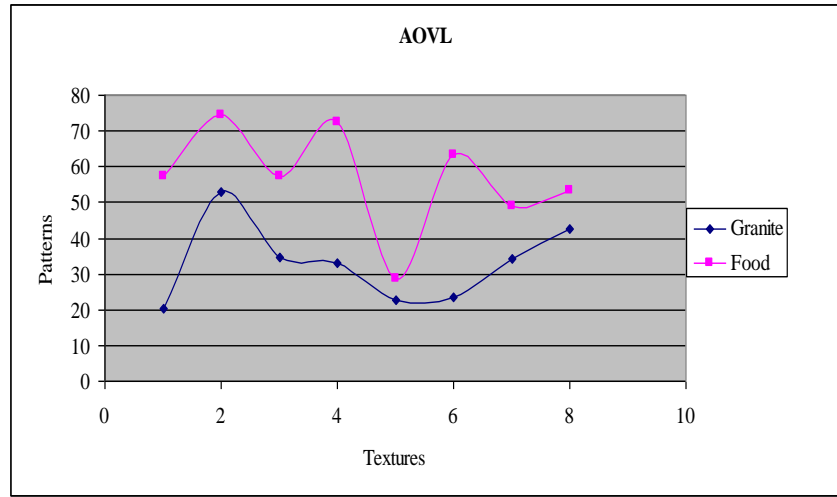

Fig. 8 Classification of Granite and Food Textures by AOVL

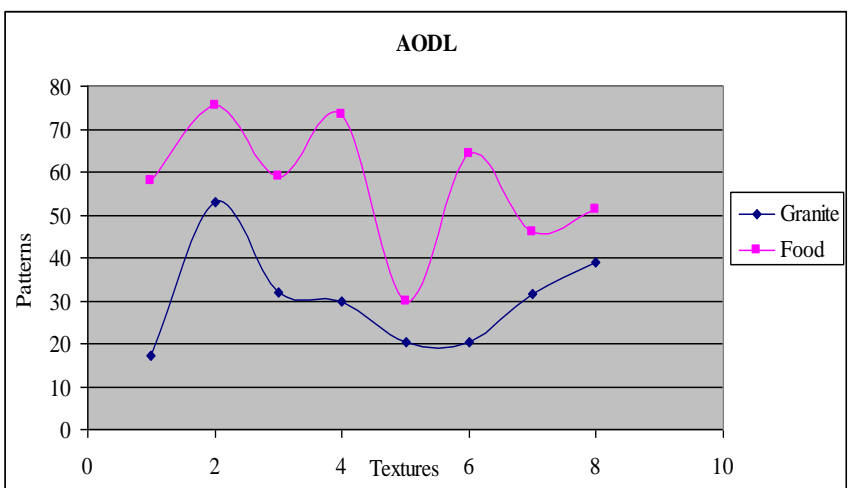

Fig. 9 Classification of Granite and Food Textures by AODL

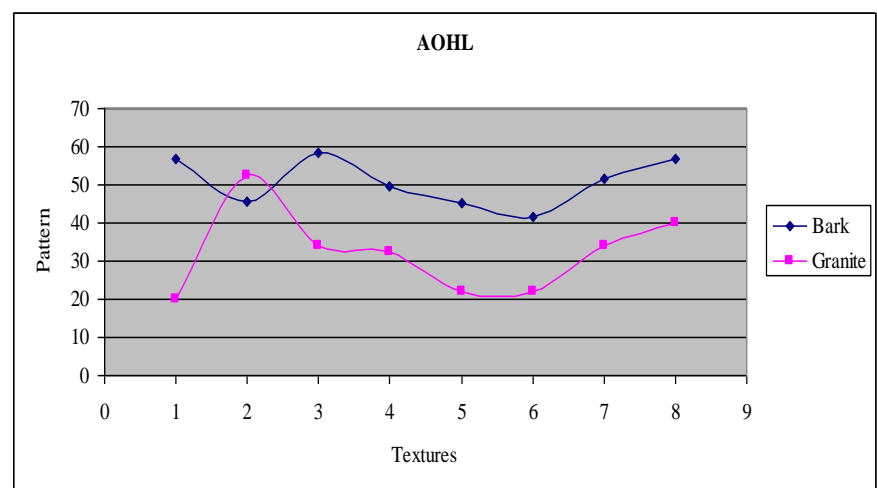

Fig. 10 Classification of Bark and Granite Textures by AOHL

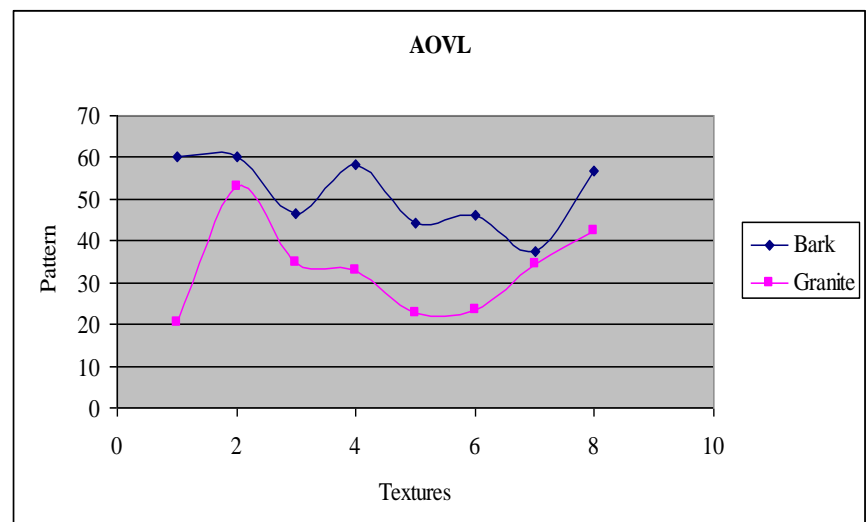

Fig. 11 Classification of Bark and Granite Textures by AOVL 


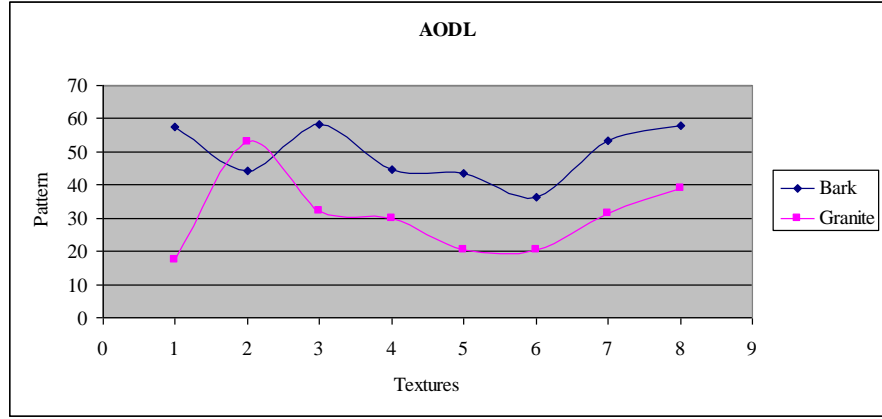

Fig. 12 Classification of Bark and Granite Textures by AODL

\section{CONCLUSIONS}

The method proposed for segment extraction uses maximum operation. Similar results are obtained by using minimum, mean and median operations for segment extraction. In this method, the threshold Te affects the size of segments in increasing order. Finally with the specified values of all parameters, the method proposed gives accurate classification of textures.

\section{Journal Papers:}

[1] J.F. Canny, "A computational approach to edge detection", IEEE Trans. Pattern Anal. Machine Intell., 8(6), 1986, 769-798, 1986Y.

[2] R. Deriche, "Using Canny's criteria to derive a recursively implemented optimal edge detector", Int. J. Computer Vision, 1(2), 1987, 187-197.

[3] M. Kass, A.Witkin and D. Terzopoulos, "Snakes: Active contour models", Int. J. Computer Vision, 1(4), 1987, 321-331.

[4] L.D. Cohen, "On active contour models and balloons", CVGIP: Image Understanding, 53(2), 1991, 211218.

[5] K. R. McCloy, "Analysis and removal of the effects of crop management practices in remotely sensed images of agricultural fields," Int. J. Remote Sens., 23, 2002, 403-416.

[6] Peder Klith Bocher and Keith R. McCloy, "The Fundamentals of Average Local Variance-Part I: Detecting Regular Patterns", IEEE Transactions on Image Processing, 15(2), 2006, 300-310.

[7] Dr. V. Vijaya Kumar, B. Eswar Reddy, U.S.N. Raju, "A Measure of Pattern Trends on Various Types of Preprocessed Textures", IJCSNS International Journal of Computer Science and Network Security, 7(8), 2007, 253-257.

[8] Modestino, J.W. and R.W. Fries, "Edge Detection in Noisy Images Using Recursive Digital Filtering", Comput. Graph. Image proc., 6, 1997, 409-433.

[9] http://www.akarmarble.com

[10] Idrissi Sidi Yassine and Samir Belfkih, "Texture Image Segmentation using a New Descriptor and Mathematical Morphology," International Arab Journal of Information Technology, 10(2), 2013, 204208.

[11] Bo-Young Park, Hyo-Hun Kim, and Byung-Woo Hong, "A Multilabel Texture Segmentation Based on Local Entropy Signature," Mathematical Problems in Engineering, 13, 2013, 1-6. 